\title{
Understanding the structure of the proton: From HERA and Tevatron to LHC
}

\author{
Laurent SCHOEFFEL* \\ CEA Saclay, Irfu/SPP, France \\ E-mail: laurent.schoeffelecea.fr
}

Understanding the fundamental structure of matter requires an understanding of how quarks and gluons are assembled to form hadrons and of the structure of the protons which are the colliding particles at LHC. The arrangement of quarks and gluons inside nucleons can be probed by accelerating electrons, hadrons or nuclei to precisely controlled energies, smashing them into a target nucleus and examining in detail the final products. The LHC physics program is rich and has been widely described. It encompasses the searches for new particles up to masses of several $\mathrm{TeV}$, including the elucidation of electroweak symmetry breaking and the possible observation of new symmetries at higher scales, and precision measurements of fundamental parameters in the electroweak and strong gauge sectors. Obviously, this program requires a precise understanding of the structure of the proton in terms of quarks and gluons, obtained from HERA and Tevatron. However, the knowledge on parton distribution functions (PDFs) is still limited for many aspects of LHC physics and the discovery potential is thus reduced. In this proceeding, we show on one example that it is possible to find some observables less sensitive to PDF uncertainties to probe with a high efficency new physics beyond the standard model.

European Physical Society Europhysics Conference on High Energy Physics

July 16-22, 2009

Krakow, Poland

* Speaker. 


\section{Introduction}

At LHC, most measurements will be limited by systematic uncertainties. Experimental systematics can be reduced in ratios of quantities. In Ref. [1], we build appropriate ratios, for which the sensitivity on theoretical uncertainties has been reduced compared to individual cross sections. The total error can then be reduced at a few percent level, showing that precision measurements at hadron colliders are possible. We illustrate this recent work on one example.

\section{Drell-Yan production cross sections ratio}

With millions of $Z / \gamma^{*}$ produced with $1 \mathrm{fb}^{-1}$ of LHC data, the statistical error on the DrellYan production cross section is expected to be smaller than the percent. The limitation comes from systematics, among which a large error is due to PDF, around 6-8\%, even at high mass $\mathrm{M}>200 \mathrm{GeV} / \mathrm{c}^{2}$. The idea is to exploit the $Z / \gamma^{*}$ mass and rapidity spectrum and to make ratios of cross sections when the initial quarks have the same kinematics.

Let us consider a quark and anti-quark that produce a $Z$ boson with a rapidity $y$. The momentum fractions of these partons are $x_{1}=M_{Z} / \sqrt{s} \cdot e^{-|y|}$ and $x_{2}=M_{Z} / \sqrt{s} \cdot e^{+|y|}$. But these momentum fractions can also be encountered in other $\gamma^{*} / Z$ processes. Symetric $q \bar{q}$ collisions with two partons carrying the momentum fraction $x_{1}$ can produce $\gamma^{*} / Z$ particles with the invariant mass $m=\sqrt{x_{1} x_{1} s}=M_{Z} \cdot e^{-|y|}$ and rapidity of 0 . In the same way, symetric $q \bar{q}$ collisions with two partons carrying the momentum fraction $x_{2}$ can produce $\gamma^{*} / Z$ particles with the invariant mass $M=\sqrt{x_{2} x_{2} s}=M_{Z} \cdot e^{+|y|}$ and rapidity of 0 . In other words, the same quark momenta have been found in three cross sections : $\sigma_{Z / \gamma^{*}}\left(M_{Z}, y\right), \sigma_{Z / \gamma^{*}}(m, y=0)$ and $\sigma_{Z / \gamma^{*}}(M, y=0)$ where $y=\ln M / M_{Z}$ and $m=M_{Z}^{2} / M$. Uncertainties on quark kinematics could be reduced in the following ratio, involving these three cross sections :

$$
R(M)=\frac{\sigma(m, y=0) \cdot \sigma(M, y=0)}{\sigma^{2}\left(M_{Z}, y\right)}
$$

where $y=\ln M / M_{Z}$ and $m=M_{Z}^{2} / M$.

With only one quark flavour and with scale invariance, the PDF completely cancel and so their uncertainties. This is no longer valid in the real case but the prediction of $R(M)$ is still more precise than the high mass Drell-Yan cross section $\sigma(M, y=0)$. Fig. 1 shows how these errors vary for different $Z / \gamma^{*}$ invariant masses. The PDF uncertainties can be reduced by more than a factor two, leading to a higher sensitivity to non-Standard Model processes.

An example of new physics sensitivity is shown on Fig. 1. Pseudo-measurement of $\sigma_{Z / \gamma^{*}}(M, y=$ 0 ) and $R(M)$, including a $2 \mathrm{TeV}$ SSM $Z^{\prime}$ are compared to the Standard Model predictions. The statistical and PDF-induced uncertainties are also displayed. A measurement of $R(M)$ shows a sensitivity of a $2 \mathrm{TeV} \mathrm{SSM} Z^{\prime}$ since $M>200 \mathrm{GeV} / c^{2}$, while in a $\sigma_{Z / \gamma^{*}}(M, y=0)$ cross section analysis, no significant deviation is seen, except for $M>600 \mathrm{GeV} / \mathrm{c}^{2}$. Thus, it seems possible to explore a larger range of $Z^{\prime}$ models, that may not be discovered by direct peak searches like non-resonant or wide $Z^{\prime}$. 


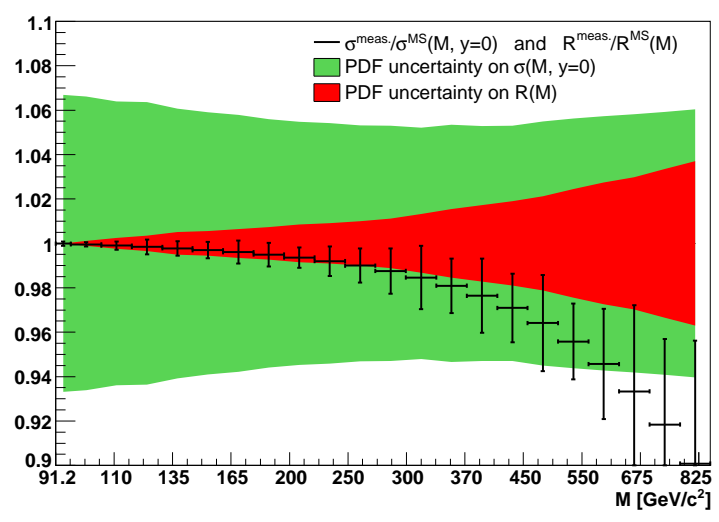

Figure 1: Pseudo-measurement to Standard Model prediction ratios of $\sigma_{Z / \gamma^{*}}(M, y=0)$ or $R(M)$ with statistical error bars. The pseudo-measurements use $30 \mathrm{fb}^{-1}$ of LHC data, and a 2 TeV SSM $Z^{\prime}$ have been added in the simulation. The central values of these two measurement are the same and the statistical uncertainties are very close, so only one set of error bars is shown. The uncertainty bands due to PDF on $\sigma_{Z / \gamma^{*}}(M, y=0)$ and $R(M)$ are also represented.

This method has other advantages. If a $Z^{\prime}$ peak is observed, this ratio of cross sections can be used to measure the $\gamma^{*} / Z / Z^{\prime}$ interference term at lower masses, in order to give additional constrains to the underlying $Z^{\prime}$ model. Finally, this method can be applied to any $s$-channel processes like $W^{ \pm}$production. $W^{\prime}$ searches or $s$-channel single-top cross sections can be normalized to $W^{ \pm} \rightarrow l v$ to obtain more precise measurements.

\section{Conclusion}

Parton momentum density distributions are important ingredients in the calculation of high energy hadron-hadron and lepton-hadron scattering cross sections. In these calculations the cross sections are written as a convolution of the parton densities and the elementary cross sections for parton-parton or lepton-parton scattering. In Ref. [1], it is shown that the vision of the proton we have at present was definitely improved with the recent data from HERA and Tevatron but still suffers from large uncertainties at low or high $x$, with significant impact on LHC plysics. No doubt that the understanding of the proton structure will be further improved at LHC, and new observables less sensitive to PDF uncertainties can be used to disantangle in a better way the PDF effects from the ones due to new physics. In this proceeding, we have presented one particular observable, defined as a ratio of Drell-Yan cross sections, for which the sensitivity on theoretical uncertainties has been reduced compared to individual cross sections. Then, this ratio shows a sensitivity of a $2 \mathrm{TeV}$ SSM $Z^{\prime}$ since $M>200 \mathrm{GeV} / c^{2}$, where $M$ is the invariant mass of the DY system. In contrast, for a $\sigma_{Z / \gamma^{*}}(M, y=0)$ cross section analysis, no significant deviation is seen, except for $M>600 \mathrm{GeV} / \mathrm{c}^{2}$.

\section{References}

[1] M. Boonekamp, F. Chevallier, C. Royon and L. Schoeffel, arXiv:0902.1678 [hep-ph]. 